\title{
Estimation of $Q$ factors from reflection seismic data for a band-limited and stabilized inverse $Q$ filter driven by an average- $Q$ model
}

\author{
Zengbao Chen ${ }^{\mathrm{a}, \mathrm{b}}$, Xiaohong Chen ${ }^{\mathrm{a}, \mathrm{b}}$,Yanghua Wang ${ }^{\mathrm{c}}$ and Jingye $\mathrm{Li}^{\mathrm{a}} \mathrm{b}$ \\ a State Key Laboratory of Petroleum Resources and Prospecting, China University of Petroleum, \\ Beijing, 102249, China. \\ b National Engineering Laboratory for Offshore Oil Exploration, China University of Petroleum, \\ Beijing, 102249, China. \\ c Centre for Reservoir Geophysics, Department of Earth Science and Engineering, Imperial College \\ London, SW7 2BP, UK.
}

\begin{abstract}
Reliable $Q$ estimation is desirable for model-based inverse $Q$ filtering to improve seismic resolution. On the one hand, conventional methods estimate $Q$ from the amplitude spectra or frequency variations of individual wavelets at different depth (or time) levels, which is vulnerable to the effects of spectral interference and ambient noise. On the other hand, most inverse $Q$ filtering algorithms are sensitive to noise, in order not to boost them, sometimes at the expense of degrading compensation effect. In this paper, the average- $Q$ values are obtained from reflection seismic data based on the Gabor transform spectrum of a seismic trace. We transform the 2-D time-variant frequency spectrum into the 1-D spectrum, and then estimate the average- $Q$ values based on the amplitude attenuation and compensation functions, respectively. Driven by the estimated average- $Q$ model, we also develop a modified inverse $Q$ filtering algorithm by incorporating a time-variant bandpass filter (TVBF), whose high cutoff frequency follows a hyperbola along the traveltime from a specified time. Finally, we test this modified inverse $Q$ filtering algorithm on synthetic data and perform the $Q$ estimation procedure on a real reflection seismic data, followed by applying the modified inverse $Q$ filtering algorithm. The synthetic data test and the real data example demonstrate that the algorithm driven by average- $Q$ model may enhance the seismic resolution, without degrading the signal-to-noise ratio.
\end{abstract}

Keywords: Attenuation compensation, $Q$ estimation, Inverse $Q$ filtering, High resolution, Time-variant band-pass filter, Gabor transform

\section{Introduction}

In seismic exploration, attenuation properties are used as an important attribute to indicate hydrocarbon in reservoir characterization (Parra and Hackert, 2002; Castagna et al., 2003; Dvorkin and Mavko, 2006). In data processing and imaging, a reliable earth $Q$ model is also a prerequisite for inverse $Q$ filtering to enhance seismic resolution. Vertical seismic profiling (VSP) or crosshole data is suitable for $Q$ estimation as direct arrivals are recorded at two different depths or different reflections are recorded at a single depth (Raikes and White, 
1984; Turgut and Yamamoto, 1990; Tonn, 1991). However, such precious data are not always available in some areas, due to the high acquisition cost. The estimation of $Q$ factors from surface reflection seismic data has recently been developed (Dasgupta and Clark, 1998; Zhang and Ulrych, 2002; Wang, 2004; Reine et al., 2012a, b).

The accuracy of $Q$ estimation from seismic data strongly depends on the frequency bandwidth and signal-to-noise ratio of the data (White, 1992). Conventional $Q$ estimation, such as spectral-ratio (Spencer et al., 1982; Tonn, 1991) or frequency-shift method (Quan and Harris, 1997; Zhang and Ulrych, 2002), compares two individual wavelets at different depth (or time) levels. However, the spectral interference from the adjacent reflections limits the application of the conventional methods. In order to reduce the presence of spectral interference, Reine et al. (2009) made use of a variable-window time-frequency transform for spectral measurements. To measure the attenuation, Reine et al. $(2012 \mathrm{a}, \mathrm{b})$ introduced an inversion scheme by operating simultaneously in the frequency and traveltime-difference coordinates to minimize the effects of the remaining interference, and by operating along traces of constant horizontal slowness through the use of $\tau-p$ transform under an assumption of a 1-D medium.

Instead of analysing individual wavelets, Wang (2004) proposed two $Q$-analysis methods based on the amplitude attenuation and compensation functions, respectively. The Gabor transform (Gabor, 1946) of a seismic trace, which reveals the localized time and frequency characteristics simultaneously, is transformed into a 1-D spectrum. The 1-D spectrum with respect to frequency-time product is used to estimate the average- $Q$ value. In absorptive media, the width of a seismic wavelet increases with traveltime due to the velocity dispersion. To allow the window to enclose an entire seismic wavelet at each time location, spectrum analysis need to use a time-variant window function, i.e., the width of the window function increases with the traveltime. In Gabor transform, we use a linear variable-window to accommodate the time-variant seismic wavelet and thereby get a more accurate 1-D spectrum from the 2-D time-variant frequency spectrum.

The wavelet transform (Chakraborty and Okaya, 1994, 1995) is another useful tool for the seismic spectrum analysis. Orthogonal and complete wavelet bases can be constructed to perform multi-resolution analysis of signals (Mallat, 1999). However, it is important to note that the orthogonality and completeness of wavelet bases are not required for the application to measure the 1-D amplitude attenuation. Both Gabor transform and the wavelet transform can be used for the spectrum analysis.

Once the explicit $Q$ model is obtained, we could apply inverse $Q$ filtering to eliminate the dissipation effect and velocity dispersion separately or simultaneously. Hale (1982) proposed an inverse $Q$ filtering method based on the Futterman model, approximately compensating the high frequency content by a series expansion. In order to further improve efficiency, Hargreaves and Calvert (1991) developed an inverse $Q$ filter approach akin to the Stolt (1978) wavenumber-frequency domain migration. This algorithm can efficiently correct for the phase distortion from velocity dispersion based on wavefield extrapolation, but neglects the amplitude effect. Wang (2002) proposed a stable and efficient approach of inverse $Q$ filtering, applying amplitude compensation and phase correction simultaneously based on stabilized wavefield downward continuation by assuming a layered earth- $Q$ model. Wang (2006) implemented an inverse $Q$ filter in the Gabor transform domain. This algorithm works for a general earth $Q$ model varying with depth or traveltime and the exact solution does not involve the amplitude operation approximation, therefore, the result of inverse $Q$ filtering is more accurate. Alternatively, Wang (2011) gave an attenuation compensation method based on inversion theory, which can be achieved by the Tikhonov regularization method solving Fredholm integral equation of the first kind. The compensation method is suitable for the 
variable $Q$ values with depth and corrects the amplitude attenuation and velocity dispersion simultaneously. However, most of these algorithms should be applied to seismic data with a high signal-to-noise ratio.

In order to avoid amplifying the ambient noise, some researchers have published their work concerning the amplitude compensation seriously. Zhang et al. (2007) gave a stable wavefield downward continuation approach to inverse $Q$ filtering for a layered earth- $Q$ model. Zhang and Ulrych (2007) formulated time-variant deconvolution as a least squares inverse problem and imposed regularization by means of Bayes' theorem, using only the forward operator and requiring no unstable inverse, however, the time-variant wavelets are required to be estimated accurately. Zhao et al. (2012) presented an approach to inverse $Q$ filtering considering the signal-to-noise ratio (SNR) in time-frequency domain, selectively compensating the amplitude in the frequency band where the SNR is larger than 1. Van der Baan (2012) proposed a promising procedure for combined dispersion and attenuation correction, by first applying dispersion corrections using phase-only inverse $Q$ filtering, followed by zero-phased time-varying Wiener deconvolution. Braga and Moaes (2013) developed an inverse $Q$ filter formulation in the wavelet domain, compensating for propagation in attenuation and dispersive media. It is implemented in a form of a damping scheme that prevents boosting ambient noise.

Due to absorption and dispersion, the time width of the seismic wavelet increases and the amplitude spectrum narrows as the wave travels. That is, a plane wave is attenuated gradually, and beyond a certain distance its amplitude has been attenuated to a level below the ambient noise level (30-70 dB), but the inverse $Q$ filter which is required to recover the signal amplifies the ambient noise. The algorithm of stabilized inverse $Q$ filtering in Gabor domain (Wang, 2006) intelligently limits the attempt to compensate the high-frequency wave component in deep level. Zhang et al. (2007) use the Gabor spectral analysis on the signals to pick gain-constrained frequencies and then estimating the corresponding gain-constrained amplitudes to control the noise amplification. Such desirable gain-constrained frequency should be time-variant and $Q$-dependent. Alternatively, a time-variant band-pass filter (TVBF) can be involved in the stabilized inverse $Q$ filtering. Pann and Shin (1976) and later Scheuer and Oldenburg (1988) implemented the TVBFs with an efficient algorithm using the theory of complex signals as a basis. Park and Black (1995) presented a new method which is able to achieve a more rapid and flexible temporal variation of the band-pass characteristics. In seismic processing, such TVBFs are designed naturally according to the earth $Q$ model and thus have a physical basis.

In this paper, we use the Gabor transform to generate a 2-D time-variant frequency spectrum, which is used for average- $Q$ estimations based on attenuation and compensation functions, respectively. The $Q$ estimations take advantage of the information from the whole seismic trace, overcoming the influence of ambient noise and reducing the effects of spectral interference to a certain extent. Then a reliable earth $Q$ model is provided for stabilized inverse $Q$ filtering by incorporating a TVBF, whose high cut-off frequency follows a hyperbola along the traveltime from a specified time. The stabilized inverse $Q$ filtering can be driven by an average- $Q$ model instead of an interval- $Q$ model, producing a desirable seismic image without boosting the ambient noise.

\section{Estimation of $\mathbf{Q}$ factors}

\section{1 estimation based on attenuation}

For a given seismic trace $u(t)$, we perform Gabor transform to generate a 2-D time- 
variant frequency spectrum $U(\tau, \omega)$, where $\tau$ is the two-way traveltime and $\omega$ is the angular frequency. We may express the amplitude attenuation of a plane wave explicitly as

$$
A(\tau, \omega)=A_{0} \exp \left(-\frac{\omega \tau}{2 Q}\right),
$$

where $A_{0}$ is the amplitude at $\tau=0$, and $Q$ is an equivalent- or average- $Q$ between $\tau=0$ and the current time $\tau$. Equation (1) can be rewritten as a linear equation

$$
\ln A^{2}(\omega \tau)=\ln A_{0}^{2}-\frac{1}{Q} \omega \tau
$$

As seismic reflection data are usually band-limited, equation (2) can be rewritten as

$$
y(\chi)=-\frac{1}{Q}\left(\chi-\chi_{a}\right),
$$

where $\chi \equiv \omega \tau$ and $y(\chi) \equiv \ln \left[A^{2}(\chi) / A^{2}\left(\chi_{a}\right)\right], A^{2}\left(\chi_{a}\right)$ is set to be the maximum power at the coordinate $\chi_{a}$, and the upper limit $\chi_{b}$ should correspond to the threshold for cutting off the small values of $y(\chi)$. The slope in equation (3) is estimated by solving the following least-squares problem within the support region $\left[\chi_{a}, \chi_{b}\right]$,

$$
J=\left\|\mathbf{y}+\frac{1}{Q} \mathbf{x}\right\|^{2} \rightarrow \min ,
$$

where $\mathbf{x}$ is the digitized variable $\chi$ and $\mathbf{y}$ is the discrete data set $y(\chi)$. Taking derivative $d J / d Q=0$, it leads to

$$
\frac{1}{Q}=-\frac{\mathbf{x}^{\mathrm{T}} \mathbf{y}}{\mathbf{x}^{\mathrm{T}} \mathbf{x}} .
$$

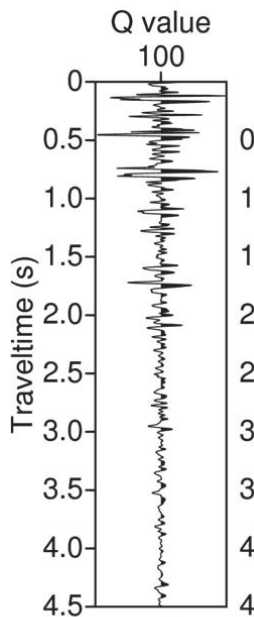

(a)

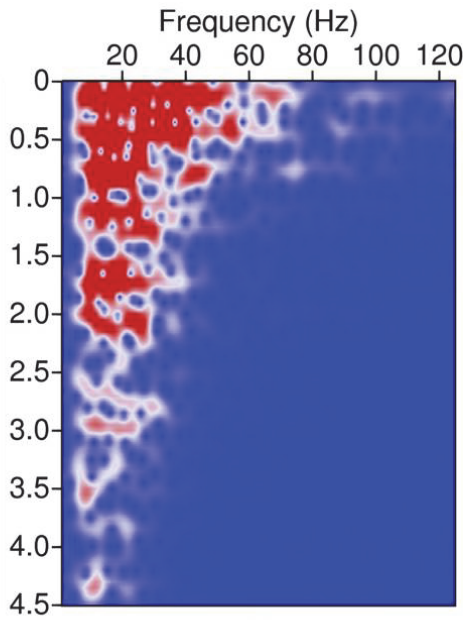

(b)

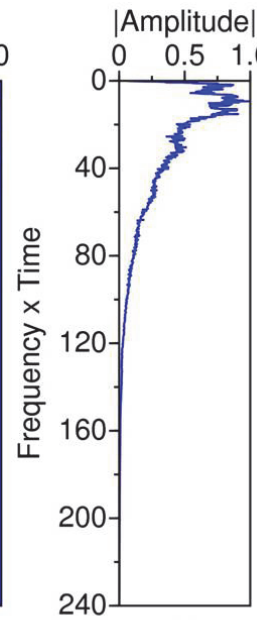

(c)

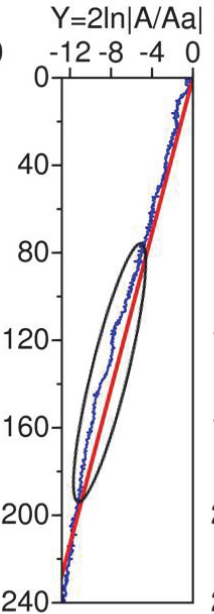

(d)

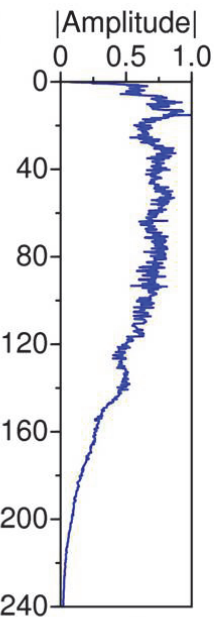

(e)

Figure 1. Seismic $Q$ estimation based on amplitude attenuation. (a) A synthetic seismic attenuated trace with known $Q$ value, $Q=100$. (b) The Gabor transform spectrum with uniform-window. (c) 1-D normalized amplitude spectrum with respect to frequency-time product before inverse $Q$ filtering. (d) Estimating $Q$ from 1-D logarithmic spectrum, where the red line corresponds to the estimated $Q$ value, $Q=111.7$. (e) 1-D amplitude spectrum with respect to frequency-time product after inverse $Q$ filtering. 
Figure 1 demonstrates the implementation of $Q$ estimation. Figure $1 \mathrm{a}$ is an attenuated trace with a known $Q$ value which is 100 , and the 2-D spectrum (Figure 1b) is produced by Gabor transform with uniform analysis window, and the attenuation characteristics at different depth (or time) levels are clearly visible. However, the support width of the analysis window needs to be increased with traveltime in order to accommodate the time-variant seismic wavelet. The 1-D amplitude spectrum (Figure 1c) decreases monotonically along axis $\chi$ and the average- $Q$ can be estimated from 1-D logarithmic spectrum by linear regression according to equation (3). The deviation in the oval (Figure 1d) is due to the effect of spectral interference. The 1-D amplitude spectrum after inverse $Q$ filtering is shown in Figure 1e.

We conduct the $Q$ estimation procedure from $2.0 \mathrm{~s}$ with an increment of $0.5 \mathrm{~s}$, and the results estimated based on attenuation using uniform and linear-variable Gabor analysis windows respectively are displayed in Table 1 . Compared to the true $Q$ value 100, the results indicate that the linear variable-window Gabor transform may generate a more reasonable 2D time-variant frequency spectrum of an attenuated seismic trace.

Table 1: The $Q$ values estimated based on attenuation using uniform and linearvariable Gabor analysis windows respectively.

\begin{tabular}{ccc}
\hline Time $(\mathrm{s})$ & $\mathrm{Q}_{1}$ & $\mathrm{Q}_{2}$ \\
\hline $0-2.0$ & 100.0 & 97.5 \\
$0-2.5$ & 103.0 & 102.9 \\
$0-3.0$ & 107.3 & 105.8 \\
$0-3.5$ & 108.8 & 106.7 \\
$0-4.0$ & 109.2 & 106.8 \\
$0-4.5$ & 111.7 & 109.4 \\
\hline
\end{tabular}

\subsection{Q estimation based on compensation}

Given the amplitude attenuation measurement, we can use it directly to design a gain curve for compensating the amplitude spectrum. We may then use such data-driven gain curve to estimate $Q$, by fitting it with a theoretical compensation function. The gain curve is designed only from amplitude attenuation, however, once the $Q$ values are obtained explicitly, we may apply them in inverse $Q$ filter to compensate the amplitude and correct the phase simultaneously.

The 1-D amplitude attenuation measurement $A(\chi)$ is smoothed by applying a median filter and then normalized by $\bar{A}(\chi)=A(\chi) / A_{a}$. A data-driven gain curve is designed as

$$
\Lambda_{d}(\chi)=\left\{\begin{array}{ll}
1, & \chi \leq \chi_{a} \\
\frac{\bar{A}(\chi)+\sigma^{2}}{\bar{A}^{2}(\chi)+\sigma^{2}}, & \chi>\chi_{a}
\end{array},\right.
$$

where $\Lambda_{d}$ with subscript $d$ indicates that the gain function is derived directly from data. In contrast, a theoretical compensation function is expressed as

$$
\Lambda(\chi, Q)=\frac{\alpha(\chi, Q)+\sigma^{2}}{\alpha^{2}(\chi, Q)+\sigma^{2}}
$$


Table 2: The $Q$ values estimated based on compensation using uniform and linearvariable Gabor analysis windows respectively.

\begin{tabular}{ccc}
\hline Time (s) & $Q_{1}$ & $Q_{2}$ \\
\hline $0-2.0$ & 82.0 & 85.6 \\
$0-2.5$ & 90.0 & 90.3 \\
$0-3.0$ & 100.0 & 96.8 \\
$0-3.5$ & 104.0 & 99.9 \\
$0-4.0$ & 109.0 & 101.6 \\
$0-4.5$ & 112.0 & 102.8 \\
\hline
\end{tabular}

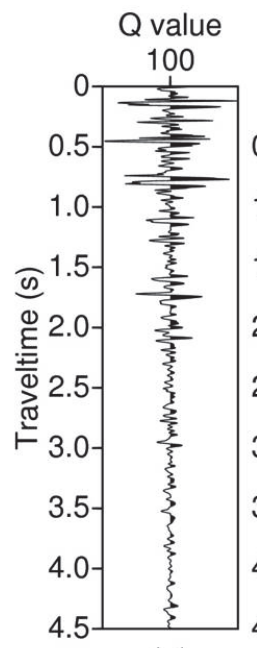

(a)

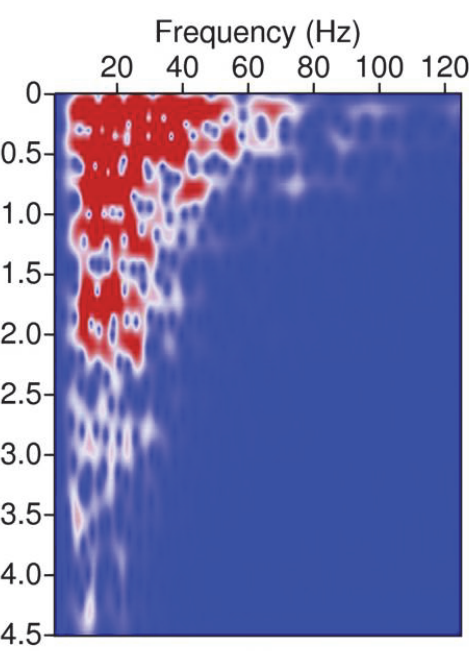

(b)

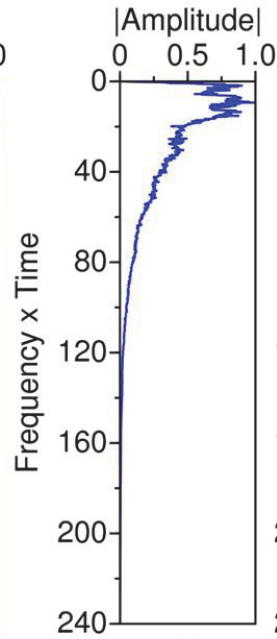

(c)

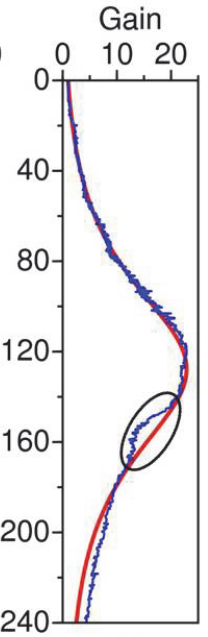

(d)

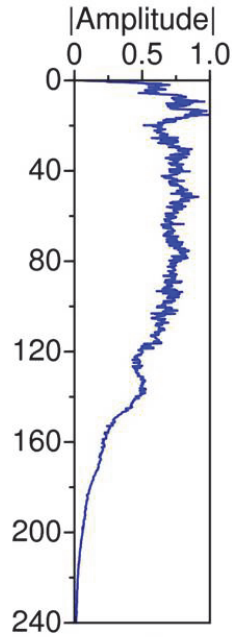

(e)

Figure 2. Seismic $Q$ estimation based on amplitude compensation. (a) A synthetic seismic attenuated trace with a known $Q$ value, $Q=100$. (b) The Gabor transform spectrum with linear variable-window. (c) 1-D normalized amplitude spectrum with respect to frequency-time product before inverse $Q$ filtering. (d) The amplitude compensation curve (blue) with the theoretical gain function (red), $Q=102.8$. (e) 1 -D amplitude spectrum with respect to frequency-time product after inverse $Q$ filtering.

where $\alpha(\chi, Q)=\exp [-\chi / 2 Q]$ and $\sigma^{2}$ is a stabilized factor which is related to the signalto-noise ratio. Finally, the $Q$ value is obtained by maximizing the correlation coefficient between the data-driven gain curve and the theoretical compensation function,

$$
\text { Coef }=\frac{\sum \Lambda_{d}(\chi) \Lambda(\chi, Q)}{\sqrt{\sum \Lambda_{d}^{2}(\chi) \sum \Lambda^{2}(\chi, Q)}} \rightarrow \max .
$$

The procedure of $Q$ estimation based on compensation is depicted in Figure 2. For the same attenuated trace (Figure 2a) as shown in Figure 1a, we use a linear variable-window Gabor transform to generate the 2-D spectrum (Figure 2b), compared with that in Figure 1b, the variations of spectral component are much more reasonable especially in the deep time. Therefore, a more accurate 1-D spectrum (Figure 2c) may be transformed to design the datadriven gain curve (Figure 2d). In this case, the estimated $Q$ value is 105.0 corresponding to the maximum correlation coefficient which is 0.9991 . The 1-D spectrum after inverse $Q$ filtering is shown in Figure 2e. This procedure is more stable and overcomes the effect of spectral interference in the oval (Figure 2d) to a certain extent.

Similarly, we show the results estimated based on compensation using uniform and linear- 
variable Gabor analysis windows respectively in Table 2 . The latter results are more accurate especially for the deep time. The deeper it is, the more reliable the estimated $Q$ value will be, as more data are included in the compensation curve.

\subsection{Interval-Q calculation}

Once we obtain a series of average- $Q$ values from the recording surface $T_{0}=0$ to different depth-time levels $T_{i}(i=1,2, \cdots)$ using either of the previous two methods, the interval- $Q$ values can be directly calculated by considering the definition of the average- $Q$ values:

$$
\left(\frac{1}{Q_{a}}\right)_{n-1}=\frac{1}{T_{n-1}} \sum_{i=1}^{n-1} \frac{\Delta T_{i}}{Q_{i}}, \quad\left(\frac{1}{Q_{a}}\right)_{n}=\frac{1}{T_{n}} \sum_{i=1}^{n} \frac{\Delta T_{i}}{Q_{i}},
$$

where $\left(1 / Q_{a}\right)_{n-1}$ and $\left(1 / Q_{a}\right)_{n}$ are the two average $1 / Q$ values between the recording surface $T_{0}=0$ and the time level $\tau=T_{n-1}$ and $\tau=T_{n}$, respectively. The interval- $Q$ value for the $n$th layer is directly given by

$$
\frac{1}{Q_{n}}=\frac{T_{n} /\left(Q_{a}\right)_{n}-T_{n-1} /\left(Q_{a}\right)_{n-1}}{T_{n}-T_{n-1}}
$$

The inverse $Q$ filtering is usually driven by an interval- $Q$ model; however, we may develop an inverse $Q$ filtering algorithm that is directly driven by the reliable average- $Q$ model.

\section{Model-based inverse $Q$ filtering}

\subsection{A review of stabilized inverse Q filtering}

Inverse $Q$ filtering is a reversal wave propagation processing that removes both absorption and dispersion effects caused by earth $Q$ filtering (Hale, 1982; Hargreaves, 1991).

In the inverse $Q$ filtering algorithm based on downward continuation, the wavefield from the recording surface $\tau_{0}=0$ to the depth-time level $\tau$, is expressed as (Wang, 2002, 2006, 2008)

$$
\begin{aligned}
U(\tau, \omega) & =U(0, \omega) \\
& \times \exp \left[\int_{0}^{\tau}\left(\frac{\omega}{\omega_{h}}\right)^{-\gamma\left(\tau^{\prime}\right)} \frac{\omega}{2 Q\left(\tau^{\prime}\right)} d \tau^{\prime}\right] \\
& \times \exp \left[i \int_{0}^{\tau}\left(\frac{\omega}{\omega_{h}}\right)^{-\gamma\left(\tau^{\prime}\right)} \omega d \tau^{\prime}\right]
\end{aligned}
$$

where $Q(\tau)$ and $\gamma(\tau)=\pi^{-1} Q^{-1}(\tau)$ are depth-dependent, the tuning parameter $\omega_{h}$ is related to the highest possible frequency of the seismic band. If we assume $Q \rightarrow \infty$ in the media, the wavefield recorded at depth-time level $\tau$ can be obtained from equation (11) as

$$
\tilde{U}(\tau, \omega)=U(0, \omega) \exp [i \omega \tau] .
$$


Both $U(\tau, \omega)$ in equation (11) and $\tilde{U}(\tau, \omega)$ in equation (12) are the wavefield recorded at the depth-time level $\tau$, but the latter is without inverse $Q$ filtering. Using $\tilde{U}(\tau, \omega)$, we rewrite equation (11) as

$$
\begin{aligned}
U(\tau, \omega) & =\tilde{U}(\tau, \omega) \\
& \times \exp \left[\int_{0}^{\tau}\left(\frac{\omega}{\omega_{h}}\right)^{-\gamma\left(\tau^{\prime}\right)} \frac{\omega}{2 Q\left(\tau^{\prime}\right)} d \tau^{\prime}\right] \\
& \times \exp \left[i \int_{0}^{\tau}\left(\left(\frac{\omega}{\omega_{h}}\right)^{-\gamma\left(\tau^{\prime}\right)}-1\right) \omega d \tau^{\prime}\right]
\end{aligned}
$$

This is the central equation for inverse $Q$ filtering in the Gabor domain and the stabilized expression is given as

$$
U(\tau, \omega)=\tilde{U}(\tau, \omega) \Lambda(\tau, \omega) \Theta(\tau, \omega),
$$

where, $\tilde{U}(\tau, \omega)$ is the Gabor transform (Gabor, 1946) of a seismic trace, representing the extrapolated wavefield at different depth-time level $\tau . \Theta(\tau, \omega)$ is the phase correction operator, which is unconditionally stable

$$
\Theta(\tau, \omega)=\exp \left[i \int_{0}^{\tau}\left(\left(\frac{\omega}{\omega_{h}}\right)^{-\gamma\left(\tau^{\prime}\right)}-1\right) \omega d \tau^{\prime}\right],
$$

and $\Lambda(\tau, \omega)$ is the stabilized amplitude compensation operator

$$
\Lambda(\tau, \omega)=\frac{\beta(\tau, \omega)+\sigma^{2}}{\beta^{2}(\tau, \omega)+\sigma^{2}},
$$

where $\beta(\tau, \omega)$ is the amplitude attenuation function

$$
\beta(\tau, \omega)=\exp \left[-\int_{0}^{\tau}\left(\frac{\omega}{\omega_{h}}\right)^{-\gamma\left(\tau^{\prime}\right)} \frac{\omega}{2 Q\left(\tau^{\prime}\right)} d \tau^{\prime}\right] .
$$

In equation (16), $\sigma^{2}$ is the stabilization factor physically related to the signal-to-noise (SNR) ratio of the seismic data. The empirical formula with respect to a specified gain limit $G_{\text {lim }}$ (in decibels) is given (Wang, 2006, 2008) as

$$
\sigma^{2}=\exp \left[-\left(0.23 G_{\text {lim }}+1.63\right)\right] \text {. }
$$

It expresses explicitly that the stabilization factor $\sigma^{2}$ controls the amplitude gain of inverse $Q$ filter. For noise-free seismic data, we can set $\sigma^{2}$ to 0.0001 in order to satisfy the requirement of stability.

Figure 3 shows a comparison of the amplitude compensation operator for the inverse $Q$ filter with the stabilized scheme. The operators are calculated with three different stabilization factors $\left(\sigma^{2}=0.001,0.01,0.1\right)$ at sample time $\tau=3 \mathrm{~s}$ for $Q=100$, respectively. The smaller the stabilization factor is, the larger the frequency corresponding to the maximum compensation amplitude is. It indicates that we should choose the stabilization factor as small as possible. 


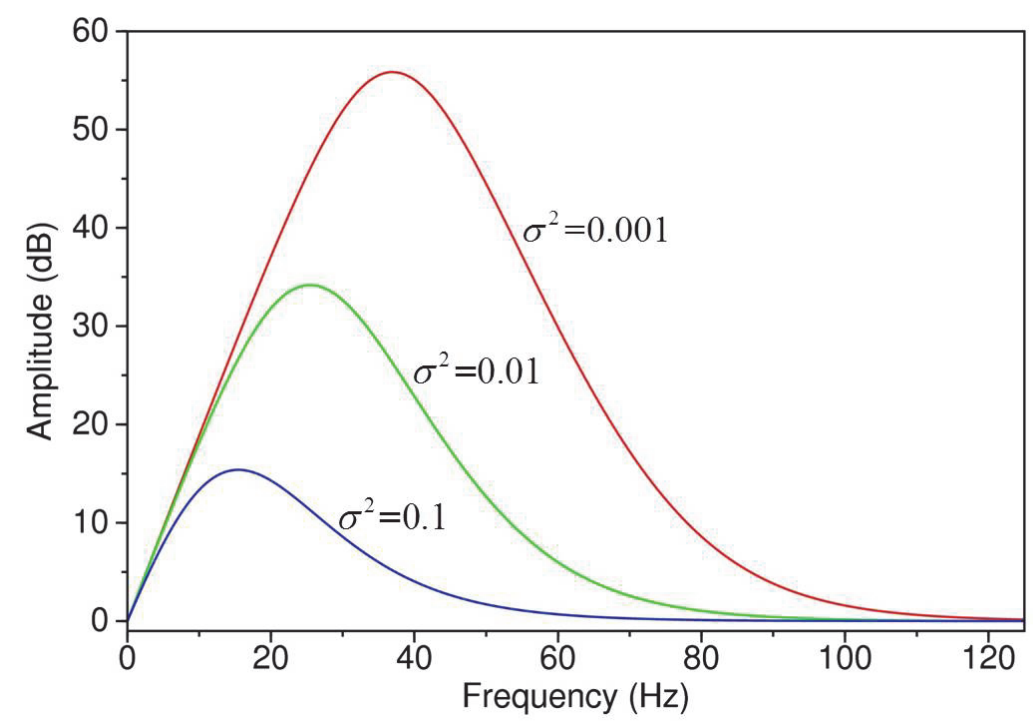

Figure 3. A comparison of amplitude compensation operators for the inverse $Q$ filter with the stabilized scheme at sample time $\tau=3 \mathrm{~s}$ for $Q=100$.

\subsection{Modified inverse Q filtering by incorporating a TVBF}

Although the interval- $Q$ values can be directly calculated from a series of average- $Q$ values (equation (10)), it may not be precise or reliable. Alternatively, we make the inverse $Q$ filter driven by a reliable average- $Q$ model instead of an interval- $Q$ model.

For $Q \gg>1$, which is appropriate for seismic data processing, we can make the approximation:

$$
\left(\frac{\omega}{\omega_{h}}\right)^{-\gamma(\tau)}-1 \approx-\gamma(\tau) \ln \left(\frac{\omega}{\omega_{h}}\right)
$$

and

$$
\left(\frac{\omega}{\omega_{h}}\right)^{-\gamma(\tau)} \approx 1
$$

Then equation (15) and (17) can be rewritten respectively as

$$
\begin{aligned}
\Theta(\tau, \omega) & \approx \exp \left[-i \int_{0}^{\tau} \ln \left(\frac{\omega}{\omega_{h}}\right) \gamma\left(\tau^{\prime}\right) \omega d \tau^{\prime}\right] \\
& =\exp \left[-i \omega \ln \left(\frac{\omega}{\omega_{h}}\right) \int_{0}^{\tau} \frac{1}{\pi Q\left(\tau^{\prime}\right)} d \tau^{\prime}\right] \\
& =\exp \left[-i \ln \left(\frac{\omega}{\omega_{h}}\right) \frac{\omega \tau}{\pi Q_{a}(\tau)}\right]
\end{aligned}
$$

and

$$
\beta(\tau, \omega) \approx \exp \left[-\int_{0}^{\tau} \frac{\omega}{2 Q\left(\tau^{\prime}\right)} d \tau^{\prime}\right]=\exp \left[-\frac{\omega \tau}{2 Q_{a}(\tau)}\right]
$$


Therefore, the amplitude compensation operator (equation 16) and the phase correction operator (equation (21)) can be determined by the reliable average- $Q$ model instead of the interval- $Q$ model.

In order to avoid amplifying the ambient noise, the stabilization factor in equation (16) should be set to a larger value, which may degrade the amplitude compensation effect. Here, we develop a stabilized inverse $Q$ filtering algorithm by incorporating a TVBF to tackle this issue. Therefore, we integrate the TVBF, denoted as $F(\tau, \omega)$, into equation (14) as

$$
U(\tau, \omega)=\tilde{U}(\tau, \omega) \Lambda(\tau, \omega) \Theta(\tau, \omega) F(\tau, \omega) .
$$

When seismic waves travel along a certain distance, the amplitude of high frequency wave component has been attenuated to a level below the ambient noise level (30-70 dB), as shown in Figure 4. We perform Gabor transform to a seismic trace to generate the 2-D Gabor spectrum $\tilde{U}(\tau, \omega)$, and in the time-frequency plane, the curves of $\tau * \omega=$ constant are hyperbolae, which means that the amplitude attenuation are at the same level. If the minimum and maximum frequencies of a bandpass filter are specified at some time, $\tau_{0}$, then we adjust them to follow a hyperbolic trajectory.

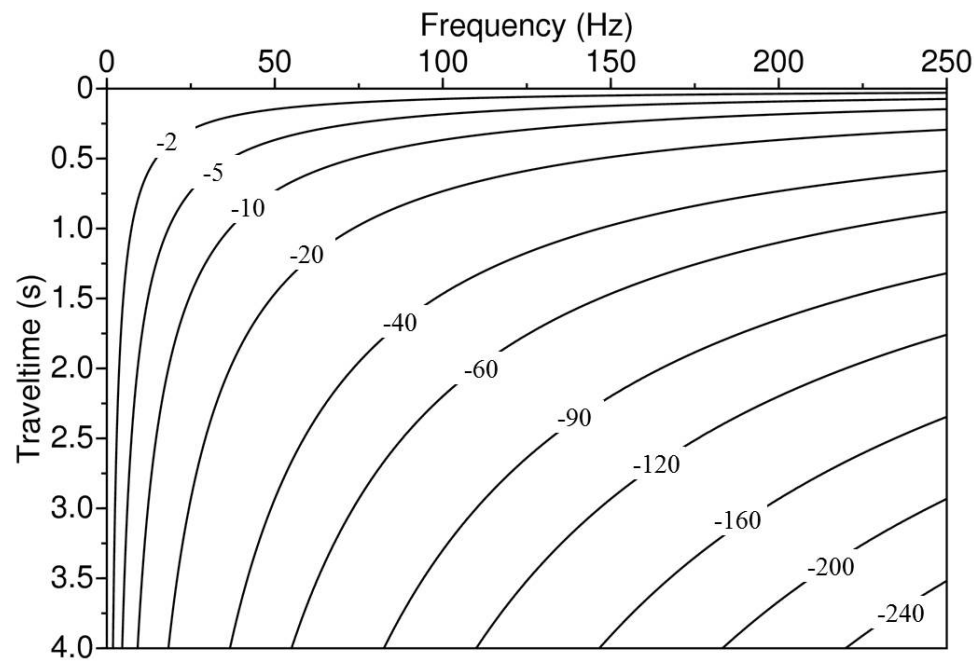

Figure 4. Contours of the constant $Q$ attenuation function (equation (22)), $Q_{a}(\tau)=100$. Contour values are expressed in decibels $(\mathrm{dB})$.

Figure 5 depicts a TVBF, which is a zero-phase filter, as the phase correction operator is unconditionally stable. At each time level, we apply a different bandpass filter, which gets a wider passband for $\tau<\tau_{0}$ and a narrower one for $\tau>\tau_{0}$. The high cutoff frequency follows a hyperbola along the traveltime from the specified time $\tau_{0}$. Note that the low and high cutoff frequencies of the bandpass should roll off to zero to avoid the Gibbs effect.

\subsection{Implementation of inverse Q filtering}

We develop the stabilized inverse $Q$ filtering in two aspects: one is that it can be driven by an average- $Q$ model instead of an interval- $Q$ model, as the reliable average- $Q$ model can be estimated directly from reflection seismic data. The other is that it may avoid amplifying the ambient noise by incorporating a TVBF, whose high cutoff frequency follows a hyperbola along the traveltime from a specified time. 


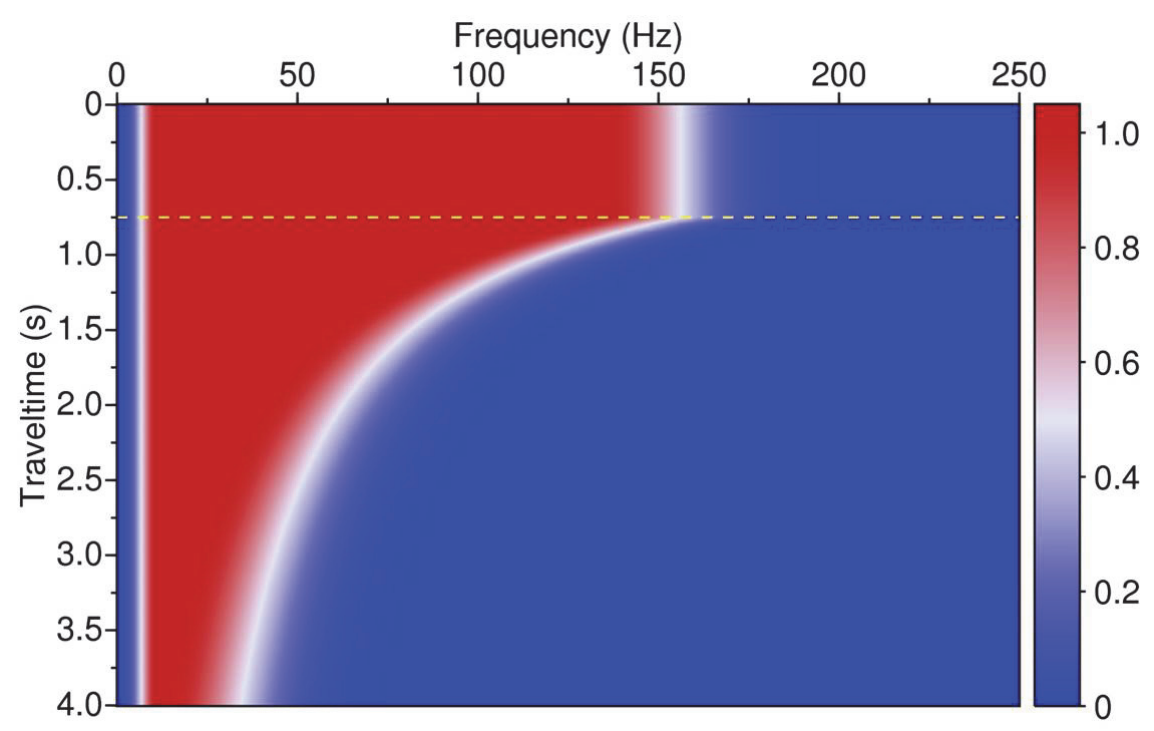

Figure 5. A depiction of a TVBF. It gets a wider passband for $\tau<\tau_{0}$ and a narrower one for $\tau>\tau_{0}$. The high cutoff frequency follows a hyperbola along the traveltime from a specified time $\tau_{0}$.

To implement the modified inverse $Q$ filtering, we use Gabor transform (Gabor, 1946) to decompose a time domain seismic trace into the time-frequency domain. Multiplying the seismic trace by a Gaussian window and then performing a Fourier transform produces a local Fourier spectrum. Repeating such a local Fourier transform iteratively for a set of window positions successively down the seismic trace, we obtain a time-variant spectrum called Gabor spectrum. We can then apply such an inverse $Q$ filter and the TVBF by modifying the Gabor spectrum before the inverse Gabor transform.

Therefore, the implementation of inverse $Q$ filtering in Gabor transform domain consists of the following three main steps:

1) for a given seismic trace $u(t)$, performing forward Gabor transform to generate the Gabor spectrum $\tilde{U}(\tau, \omega)$, which represents the extrapolated wavefield at different depth-time level $\tau$;

2) driven by the reliable average- $Q$ model, applying stabilized inverse $Q$ filtering on $\tilde{U}(\tau, \omega)$ by incorporating a TVBF to produce $U(\tau, \omega)$ according to the equation (23);

3) performing inverse Gabor transform to reproduce the seismic trace $u(t)$ in time domain.

\section{Inverse $Q$ filtering test on synthetic data}

We test the stabilized inverse $Q$ filtering algorithm on both noise-free and noise-added synthetic data.

Figure 6 displays noise-free synthetic traces and two results of applying the stabilized inverse $Q$ filtering algorithm in Gabor domain by setting the stabilization factor $\sigma^{2}$ to 0.0001 and 0.01 , respectively. Compared with the reference trace without attenuation, the stabilized inverse $Q$ filtering has recovered all the frequency components that are in principle recoverable and the phase distortion is corrected accurately. The larger stabilization factor may degrade the compensation effect especially when the $Q$ value is less than 100 .

The synthetic traces (Figure 6a) after adding Gaussian noise $(\mathrm{SNR}=10)$ are shown in Figure 7a. Compared with the result of stabilized inverse $Q$ filtering (Figure 7b), the result of stabilized inverse $Q$ filtering by incorporating a TVBF (Figure 7c) does not boost the high frequency noise by setting the same stabilization factor $\left(\sigma^{2}=0.01\right)$. 


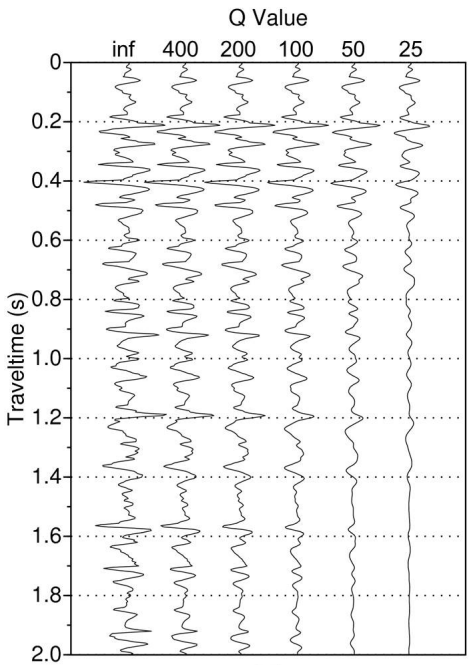

(a)

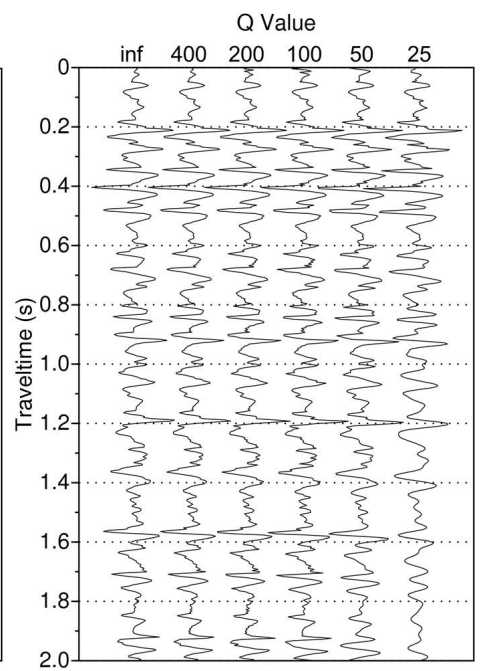

(b)

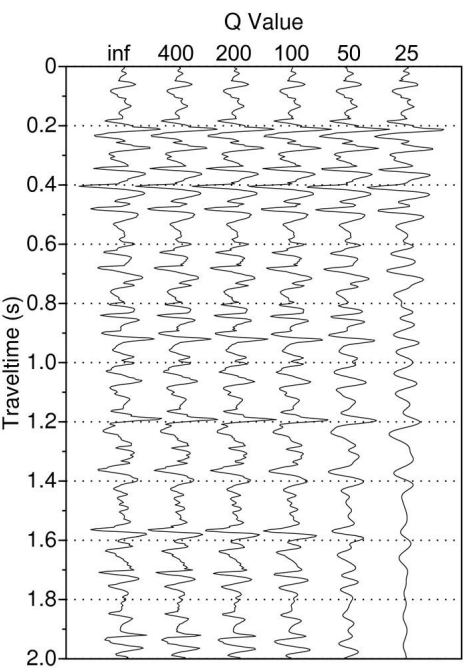

(c)

Figure 6. Noise-free synthetic traces and the results of stabilized inverse $Q$ filtering: (a) Synthetic seismic traces by applying different $Q$ values. (b) The result of stabilized inverse $Q$ filtering by setting the stabilization factor $\sigma^{2}$ to 0.0001 . (c) The result of stabilized inverse $Q$ filtering by setting the stabilization factor $\sigma^{2}$ to 0.01 . The first trace without attenuation in each figure is plotted as a reference.

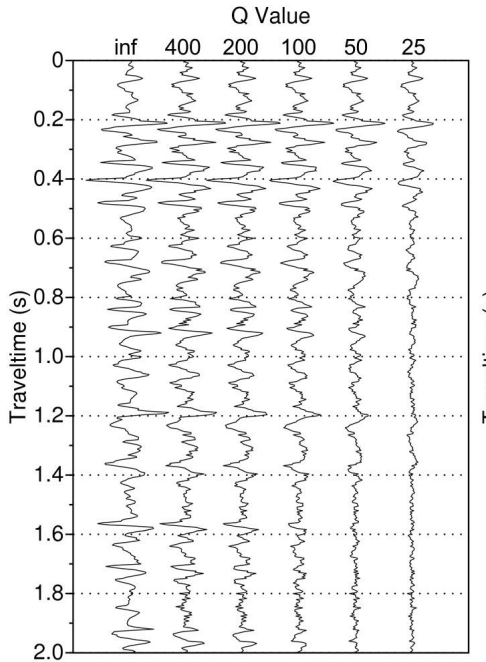

(a)

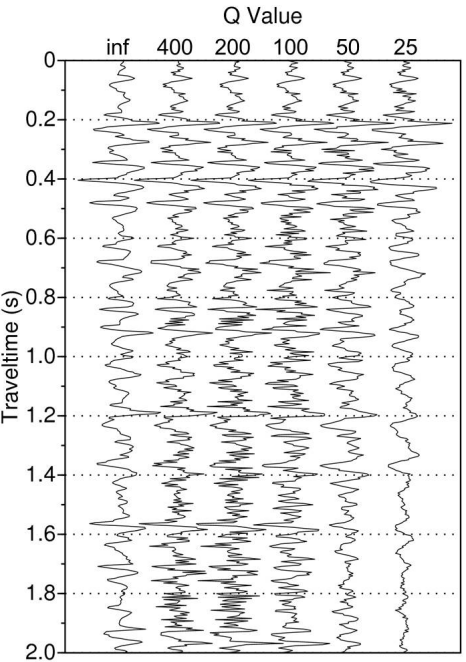

(b)

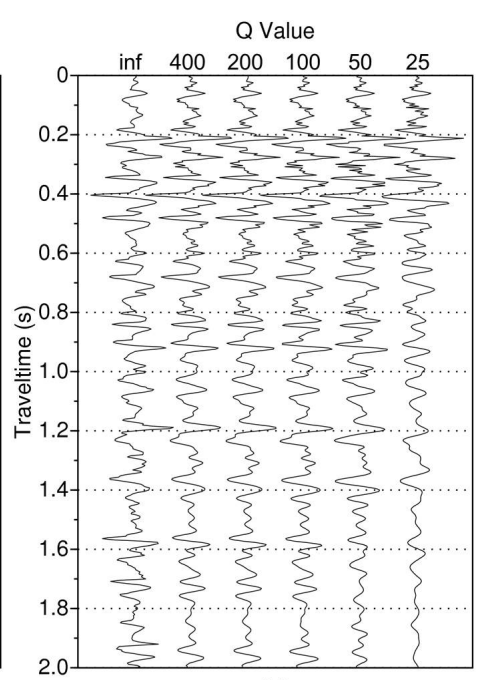

(c)

Figure 7. Noise-added synthetic traces and the results of stabilized inverse $Q$ filtering: (a) Synthetic seismic traces added with a Gaussian noise $(\mathrm{SNR}=10)$. (b) The result of stabilized inverse $Q$ filtering. (c) The result of stabilized inverse $Q$ filtering by incorporating a TVBF. The stabilization factor $\sigma^{2}$ is set to 0.01 in each case. The first trace without attenuation in each figure is plotted as a reference.

\section{A field data application example}

To demonstrate the $Q$ estimation based on the amplitude compensation, we use a real seismic reflection data set shown in Figure 8a, which is a poststack profile with a high signalto-noise ratio.

As the formations are almost horizontal from $1.0 \mathrm{~s}$, we average over all the traces to further improve the signal-to-noise ratio and then perform the linear variable-window Gabor transform to produce the 2-D spectrum (Figure 10a). This spectrum is used first to compute 
the attenuation measurement and then to derive the compensation function with respect to frequency-time product. Such data-driven gain curves are used for the average- $Q$ estimation, which is conducted from $2.5 \mathrm{~s}$ with an increment of $0.05 \mathrm{~s}$. Figures $10 \mathrm{~b}-\mathrm{i}$ display the diagnoses only at eight different times selectively. The correlation coefficient between the data-driven gain curve and the theoretical compensation function is close to 1 , which means that the average- $Q$ estimation is reliable. The interval- $Q$ values (Figure 10j) are directly calculated using equation (10) after producing a series of average- $Q$ values at different time levels, where the interval is set to $0.5 \mathrm{~s}$. The interval- $Q$ model can be used to design the inverse $Q$ filter, alternatively, we use the reliable average- $Q$ model to determine the inverse $Q$ filter.

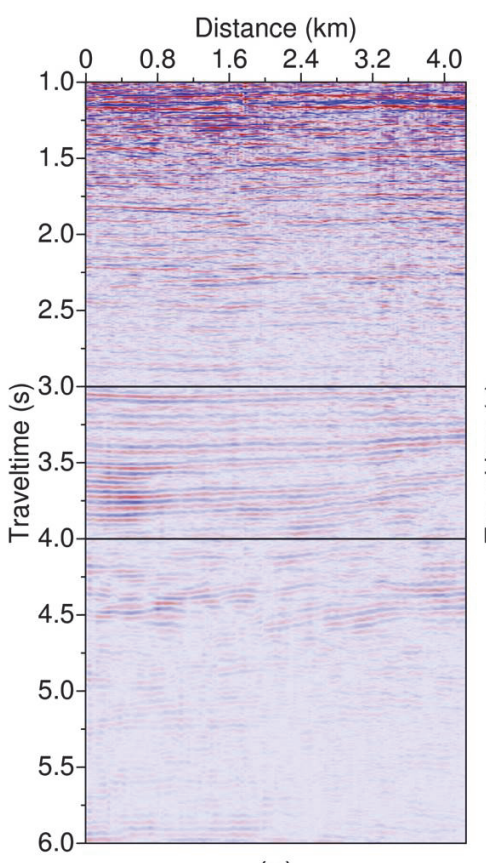

(a)

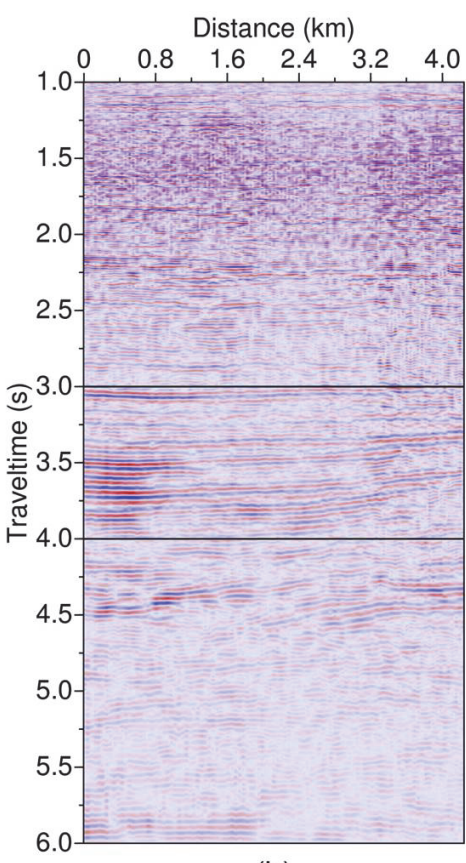

(b)

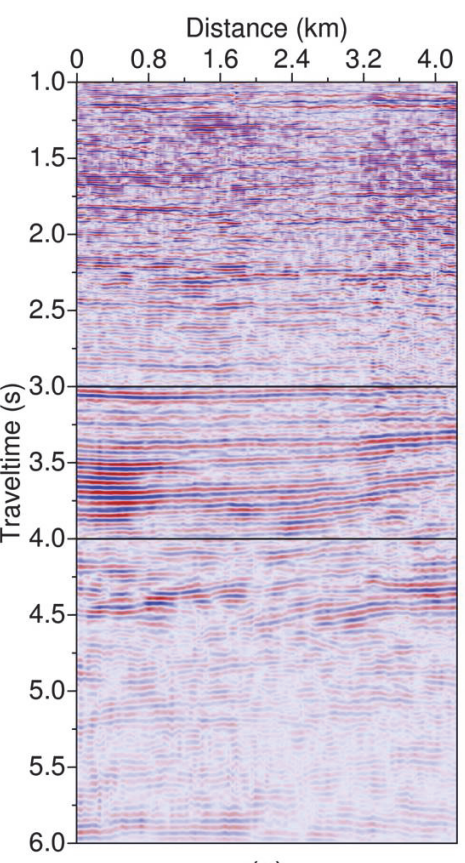

(c)

Figure 8. Real data example: (a) A stack seismic section before inverse $Q$ filtering. (b) The seismic section after modified inverse $Q$ filtering without a TVBF. (c) The seismic section after modified inverse $Q$ filtering with a TVBF. All the sections are plotted in the same scale.

In this case, we regard the depth time $1.0 \mathrm{~s}$ as a new surface and apply an inverse $Q$-filter, which is determined by the estimated average- $Q$ model, to the seismic section in Figure $8 \mathrm{a}$. The stabilization factor $\sigma^{2}$ is set to 0.001 and the results after modified inverse $Q$ filtering with and without a TVBF are plotted in Figures $8 \mathrm{~b}$ and $8 \mathrm{c}$, respectively. Apparently, the energy in deep layers is balanced with that in shallow layers. More details are revealed in the zoomed-in sections (Figure 9) between 3.0 and $4.0 \mathrm{~s}$. Changes in event times are produced by correcting for dispersion and the events become more continuous, especially in the oval areas and the section after inverse $Q$ filtering with a TVBF is much clearer than that without a TVBF.

Figure 11 compares the average amplitude spectra of the seismic profiles (Figure 8) before (black curve) and after modified inverse $Q$ filtering with (red curve) and without (blue curve) a TVBF. It shows that inverse $Q$ filter may flatten the amplitude spectrum and increase the spectral bandwidth, therefore, the seismic resolution is enhanced remarkably. By incorporating a TVBF, the inverse $Q$ filter may limit the attempt to over compensate the high-frequency wave component and thereby does not degrade the signal-to-noise $(\mathrm{S} / \mathrm{N})$ ratio. 


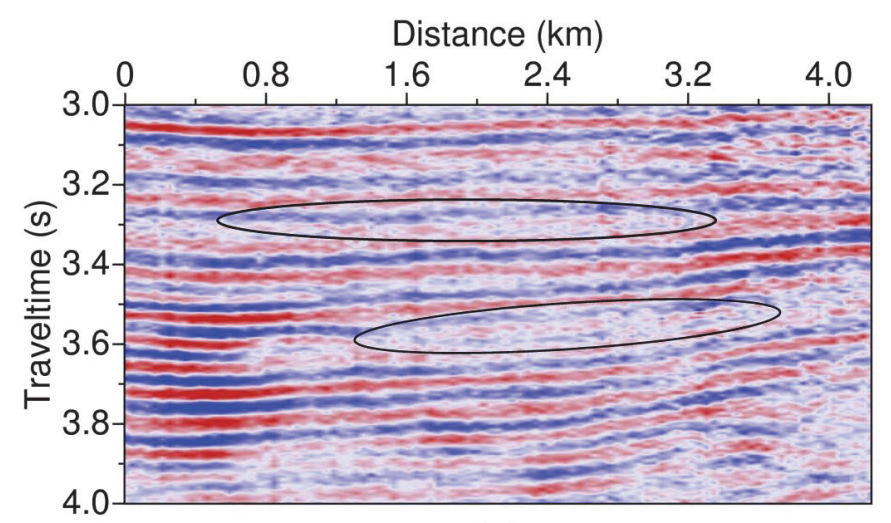

(a)

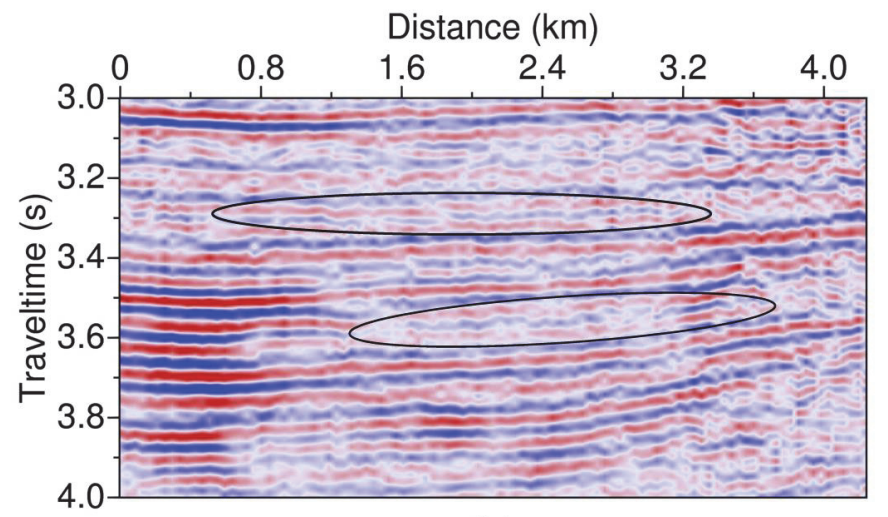

(b)

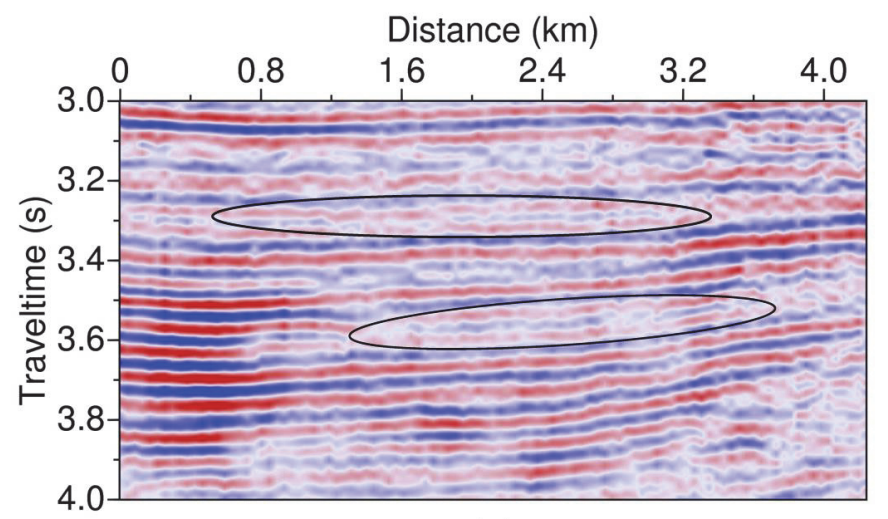

(c)

Figure 9. (a) A zoomed-in seismic section before inverse $Q$ filtering. (b) The zoomed-in section after modified inverse $Q$ filtering without a TVBF. (c) The zoomed-in section after modified inverse $Q$ filtering with a TVBF. All the sections are plotted in the same scale.

Accurate $Q$ estimation from reflection seismic data provides reliable information for $Q$ compensation. With quantitative attenuation information, it may be possible to present the true relative-amplitude variation of the seismic reflection waves, which facilitates geological and lithological interpretation.

The average- $Q$ estimation avoids spectral interference from close reflections. However, as the poststack data may be processed by time-variant frequency filtering, such as time-variant deconvolution or spectral whitening, prestack data provides information concerning structure, lithology, and material properties such as velocity and $Q$-factor. Prestack $Q$ estimation is more suitable for inverse $Q$ filtering. This method can be extended to prestack seismic 


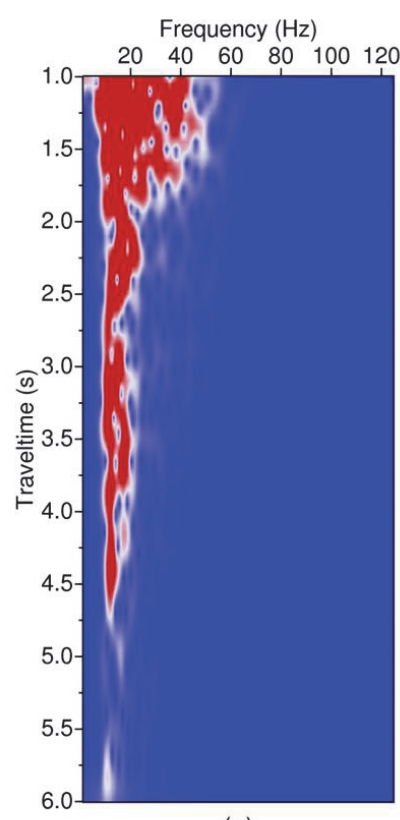

(a) (b) Time=2.5s, Qa $=38.2$

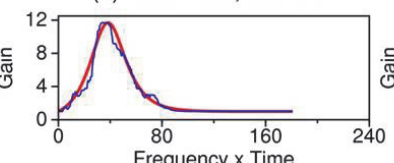

(c) Time=3.0s, Qa=44.0

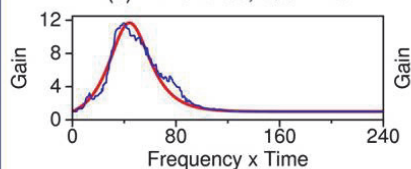

(d) Time $=3.5 \mathrm{~s}, \mathrm{Qa}=51.3$

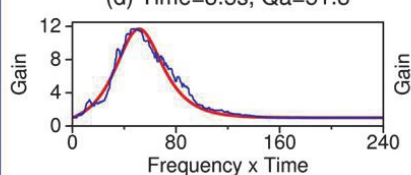

Frequency $\times$ Time
(e) Time $=4.0 \mathrm{~s}, \mathrm{Qa}=57.6$

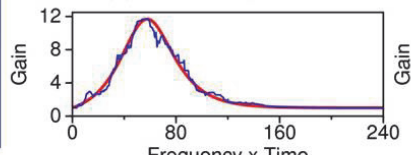

Frequency $x$ Time

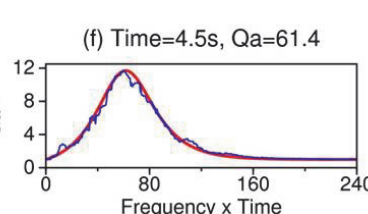

(g) Time=5.0s, Qa =63.1

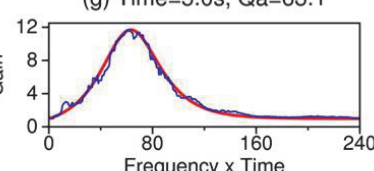

(h) Time $=5.5 \mathrm{~s}, \mathrm{Qa}=64.1$

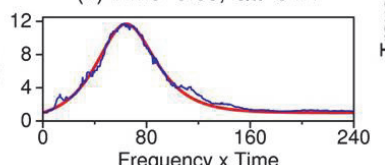

(i) Time $=6.0 \mathrm{~s}, \mathrm{Qa}=65.5$

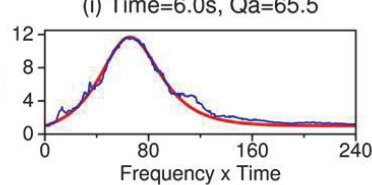

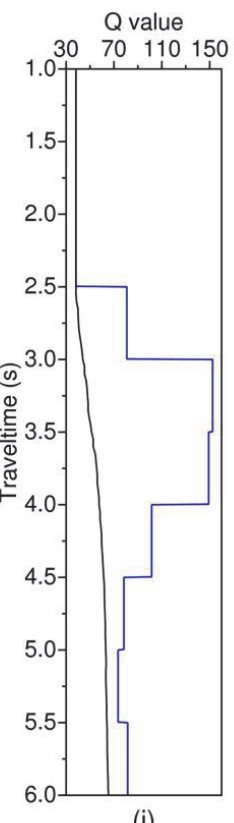

(j)

Figure 10. $Q$ estimation from reflection seismic data. (a) The Gabor transform spectrum. (b-i) The diagnosis of average- $Q$ estimations at different times. (j) The interval- $Q$ values (blue curve) corresponding to the estimated average- $Q$ values (black curve).

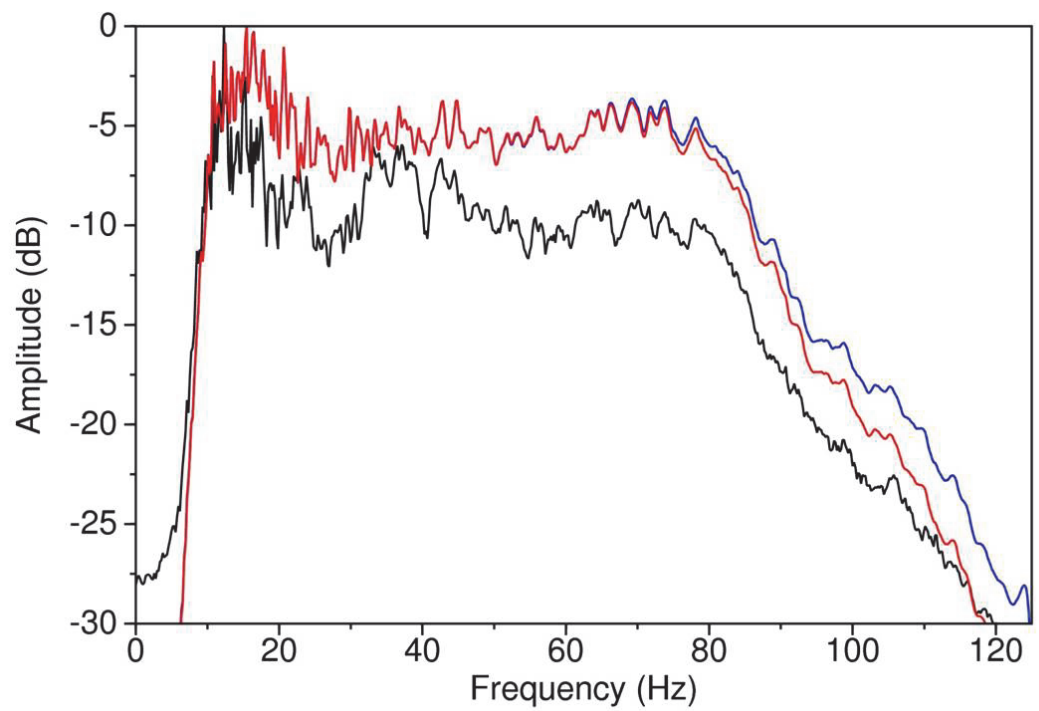

Figure 11. A comparison among the average amplitude spectra of the seismic sections before (black curve) and after modified inverse $Q$ filtering with (red curve) and without (blue curve) a TVBF. The red curve and blue curve are overlapped when the frequency is less than $70 \mathrm{~Hz}$.

processing, providing critical information for prestack $Q$ compensation. It is similar to apply the $Q$ estimation method on the prestack data after NMO.

\section{Conclusions}

Reliable average- $Q$ values can be estimated based on the Gabor transform spectrum of 
reflection seismic data. A linear variable-window Gabor transform may generate a more reasonable 2-D time-variant frequency spectrum of an attenuated seismic trace, and the interval- $Q$ values can be directly calculated after producing a series of average- $Q$ values.

The modified inverse $Q$ filtering by incorporating a TVBF is robust for noisy data. Compared with the stabilized inverse $Q$ filtering without a TVBF, it does not boost the ambient noise by setting a smaller stabilization factor. The synthetic data test illustrates that the algorithm may enhance the seismic resolution, without degrading the signal-to-noise ratio.

The field data application example demonstrates that the compensation-based $Q$ estimation is stable and effective to provide a reliable average- $Q$ model for inverse $Q$ filtering, producing a desirable seismic image with high resolution.

\section{Acknowledgements}

This research is supported by the National Natural Science Foundation of China (Grant No. U1262207, 41074098) and the National Science and Technology of Major Projects of China (No. 2011ZX05023-005-005). We are also grateful to the sponsors of the Centre for Reservoir Geophysics, Imperial College London, for supporting this research. The first author would like to thank China Scholarship Council for their financial support to study at Imperial College London.

\section{References}

Braga, I.L.S., Moraes, F.S., 2013. High-resolution gathers by inverse $Q$ filtering in the wavelet domain. Geophysics 78 (2), V53-V61.

Castagna, J.P., Sun, S., Siegfried, R.W., 2003. Instantaneous spectral analysis Detection of low-frequency shadows associated with hydrocarbons. The Leading Edge 22 (2), 120-127.

Chakraborty, A., Okaya, D., 1994. Application of wavelet transform to seismic data. 64th Annual International Meeting., SEG, Expanded Abstracts, pp. 725-728.

Chakraborty, A., Okaya, D., 1995. Frequency-time decomposition of seismic data using wavelet based methods. Geophysics 60 (6), 1906-1916.

Dasgupta, R., Clark, R., 1998. Estimation of $Q$ from surface seismic reflection data. Geophysics 63 (6), 2120-2128.

Dvorkin, J.P., Mavko, G., 2006. Modeling attenuation in reservoir and nonreservoir rock. The Leading Edge 25 (2), 194-197.

Gabor, D., 1946. Theory of communication. Part 1: The analysis of information. Journal of the Institution of Electrical Engineers 93 (26), 429-441.

Hale, D., 1982. $Q$-adaptive deconvolution. Stanford Exploration Project Report 28, 209-231.

Hargreaves, N.D., Calvert, A.J., 1991. Inverse $Q$ filtering by Fourier transform. Geophysics 56 (4), 519-527.

Mallat, S., 1999. A wavelet tour of signal processing. Academic Press.

Pann K., Shin Y., 1976. A class of convolutional time-varying filters. Geophysics 41 (1), 2843.

Park C., Black R.A., 1995. Simple time-variant, band-pass filtering by operator scaling. Geophysics 60 (5), 1527-1535.

Parra, J.O., Hackert, C., 2002. Wave attenuation attributes as flow unit indicators. The Leading Edge 21 (6), 564-572.

Quan, Y., Harris, J.M., 1997. Seismic attenuation tomography using the frequency shift method. Geophysics 62 (3), 895-905.

Raikes, S.A., White, R.E., 1984. Measurements of earth attenuation from downhole and 
surface seismic recordings. Geophysical Prospecting 32 (5), 892-919.

Reine, C., Clark, R., Van der Baan, M., 2012a. Robust prestack $Q$-determination using surface seismic data: Part 1-Method and synthetic examples. Geophysics 77 (1), R45R56.

Reine, C., Clark, R., Van der Baan, M., 2012b. Robust prestack $Q$-determination using surface seismic data: Part 2-3D case study. Geophysics 77 (1), B1-B10.

Reine, C., Van der Baan, M., Clark, R., 2009. The robustness of seismic attenuation measurements using fixed- and variable-window time-frequency transforms. Geophysics 74 (2), WA123-WA135.

Scheuer T., Oldenburg D.W., 1988. Aspects of time-variant filtering. Geophysics 53 (11), 1399-1409.

Spencer, T., Sonnad J., Butler T., 1982. Seismic $Q$ stratigraphy or dissipation. Geophysics 47 (1), 16-24.

Stolt, R., 1978. Migration by Fourier transform. Geophysics 43 (1), 23-48.

Tonn, R., 1991. The determination of the seismic quality factor $Q$ from VSP data: A comparison of different computational methods. Geophysical Prospecting 39 (1), 1-27.

Turgut, A., Yamamoto, T., 1990. Measurements of acoustic wave velocities and attenuation in marine sediments. The Journal of the Acoustical Society of America 87 (6), 2376-2383.

Van der Baan, M., 2012. Bandwidth enhancement: Inverse $Q$ filtering or time-varying Wiener deconvolution?. Geophysics 77 (4), V133-V142.

Wang, S., 2011. Attenuation compensation method based on inversion. Applied Geophysics $8(2), 150-157$.

Wang, Y., 2002. A stable and efficient approach of inverse $Q$ filtering. Geophysics 67 (2), 657-663.

Wang, Y., 2004. $Q$ analysis on reflection seismic data. Geophysical Research Letters 31 (17), L17606.

Wang, Y., 2006. Inverse $Q$-filter for seismic resolution enhancement. Geophysics 71 (3), V51-V60.

Wang, Y., 2008. Seismic inverse $Q$ filtering, Blackwell Publishing, Oxford.

White, R., 1992. The accuracy of estimating $Q$ from seismic data. Geophysics 57 (11), 15081511.

Zhang C., Ulrych T.J., 2002. Estimation of quality factors from CMP records. Geophysics 67 (5), 1542-1547.

Zhang C., Ulrych T.J., 2007. Seismic absorption compensation: A least squares inverse scheme. Geophysics 72 (6), R109-R114.

Zhang, X., Han, L., Zhang, F., Shan, G., 2007. An inverse $Q$-filter algorithm based on stable wavefield continuation. Applied Geophysics 4 (4), 263-270.

Zhao, Y., Liu, Y., Li, X., Jiang, N., 2012. An approach of inverse $Q$ filtering considering time-frequency domain signal-to-noise ratio. 74th EAGE Conference \& Exhibition, Copenhagen. 